\title{
Discerning Electricity Consumption Patterns from Urban Allometric Scaling
}

\author{
R. Horta-Bernús \& M. Rosas-Casals \\ Càtedra UNESCO de Sostenibilitat \\ Universitat Politècnica de Catalunya, Campus Terrassa \\ C. Colom, 1, 08222, Barcelona, Spain \\ horta@ee.upc.edu \& rosas@mmt.upc.edu
}

\author{
S. Valverde \\ ICREA-Complex Systems Lab., PRBB \\ Universitat Pompeu Fabra \\ C. Dr. Aiguader, 88, 08003, Barcelona, Spain \\ svalver@gmail.com
}

\begin{abstract}
Allometric scaling relations are characteristic of all living organisms. Metabolic and heart rates, lifespan and many other physiological properties vary with body mass in systematic and interrelated ways, which usually take the form of a power law. Scaling laws have been recently observed also in the metabolic rate of a particular kind of living system: the city. Scaling exponents of urban indicators present a remarkable variability, mainly associated with fundamentally different underlying dynamics. In this paper allometric scaling is used to detect dissimilar behaviors in one particularly important urban indicator: electricity consumption. Different scaling relations between electricity consumption and economical and social sectors found in southern Spain region of Andalucía, indicate variability in these sectors' basal energy consumption processes. The usefulness of these findings for urban modeling is finally outlined and some practical implications are suggested.
\end{abstract}

Index Terms-allometry; electricity consumption; power law; cities;

\section{INTRODUCTION}

The word allometric refers to properties in bodies that are not isometric, but that change with size according to a particular law, being 'allometric scaling' the most generally used term. Allometric scaling has been observed mainly in biology, where metabolic rate of living organisms scales as the 3/4-power of mass over 27 orders of magnitude, from molecular and intracellular levels up to the largest organisms. Similarly, time-scales (such as lifespans and growth rates) and sizes (such as bacterial genome lengths, tree heights and mitochondrial densities) scale with exponents that are typically simple powers of $1 / 4$ [1]. Although living organisms are complex systems with hierarchical levels of organization, formed by many components and many interactions at different temporal and spatial scales, the simplicity of these relationships suggest that more fundamental principles underly much of the coarse-grained generic structure and organization of living systems. In fact the observed power law scaling can be explained by means of energy minimization models and space filling constraints. [2]

Allometric scaling has been recently observed in those particularly complex living organisms known as cities [3]. Urban agglomerations present power law scaling for much of the magnitudes that characterize their social and economical dynamics. But in contrast with biological systems, where
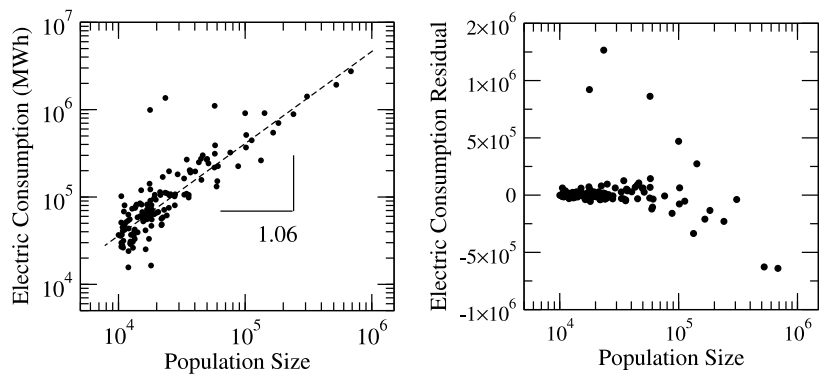

Fig. 1. Empirical allometric scaling relationships between global electricity consumption and population size. The scaling exponent is slightly superlinear, with a value of $\beta=1.06$ (left). The right plot shows the scaling of deviations $d_{i}$ from predicted values.

economies of scale are the usual outcome, urban indicators in cities present a remarkable variability. Using population, $N(t)$, as the measure of the city size at time $t$, power law scaling takes the form

$$
Y(t)=Y_{0} N^{\beta}(t)
$$

where $Y$ can denote both material resources or measures of social activity, $Y_{0}$ is a normalization constant and $\beta$ is the characteristic scaling exponent. It is precisely the variability in $\beta$ that reflects different dynamic rules at play across the urban system. Material resources such as road surface and length in electrical cables scale sublinearly, with $\beta<1$, displaying economies of scale (analogous to similar quantities in biology). On the contrary, patents, inventors, wages and similar social activity indicators scale superlinearly, with $\beta>1$, showing increasing returns with population size. Individual human needs finally, tend to scale linearly, with $\beta \approx 1$. This variability in the scaling exponent suggest unique social dynamics with important practical implications that range from recognizing limits to urban population growth to facility location modeling or defining innovative and more optimal infrastructural network designs.

Among all urban indicators, variables related with electricity generation, distribution and consumption appear as particularly meaningful ones. Electricity is the most fundamental energetic input for urban agglomerations and with the majority of people 
now living in cities [4], it can be considered not only a good proxy for their economic and social activity but also an indicator to detect future sustainable development paths and policies. Household electrical consumption scaling, for example, has been used to detect differences in the geographical location of Chinese cities, with those located in central and northern China showing sublinear scaling exponent, lower than that of coastal and biggest ones, clearly superlinear [5]. On the other hand, the analysis of the differences between scaling exponents for variables such as cable length or resistive losses, suggests the presence of a suboptimal distribution network. [3]

It seems therefore that useful information can be extracted from the scaling behavior of urban indicators in general and electricity variables in particular, since it can reveal underlying multiscale dynamics not observable from the sole analysis of individual cities. Following this path of inquiry and analysis, in this paper we present scaling relations for electrical consumption in Andalucía, the most meridional autonomous community of Spain. Our data is presented in section II, along with scaling correlations for several electricity consuming sectors. In section III we discuss our findings and section IV concludes and suggests further implications.

\section{ELECTRICITY CONSUMPTION DATA AND ALLOMETRIC SCALING}

Electricity consumption data can be retrieved from the Instituto de Estadística de Andalucía [6]. Andalucía is the most meridional autonomous region of Spain, the second largest, with an area of $87,268 \mathrm{~km}^{2}$, and the first one in population, with $8,202,220$ people. Primary and secondary sectors give occupation to almost one half of the active population. While the former is mostly dispersed, the latter is concentrated in coastal areas and in the Guadalquivir valley. Finally, tertiary sector emerges as the most economically important one, primarily centered around tourism, mainly in coastal provinces. Therefore, electrical consumption data includes cities and urban agglomerations with remarkably different climates, geography, GDP's, social dynamics and economical drivers and activities. In any case, scaling has been performed using 134 observations corresponding to 134 villages and cities in Andalucía, spanning two orders of magnitude and with data from year 2005 .

Figure 1 shows scaling behavior for the aggregated electricity consumption in Andalucian cities, together with deviations from predicted scaling (left and right plots, respectively). These have been calculated as $d_{i}=Y_{i}-Y_{0} N_{i}^{\beta}$. As we can see, $d_{i}$ grows with increasing population size. These deviations represent a normalized measure of the energetic efficiency $\left(d_{i}>0\right)$ or inefficiency $\left(d_{i}<0\right)$ of the $i-t h$ city relative to its population size $N_{i}$ and thus, they are a better comparison measure when assessing the energetic cost of any city [7]. For the global electricity consumption, although most cities remain clustered around $d_{i} \approx 0$, there is noticeable dispersion for some other cities.

However, from a non aggregated point of view we observe other patterns and different scaling exponents. Figure 2 shows
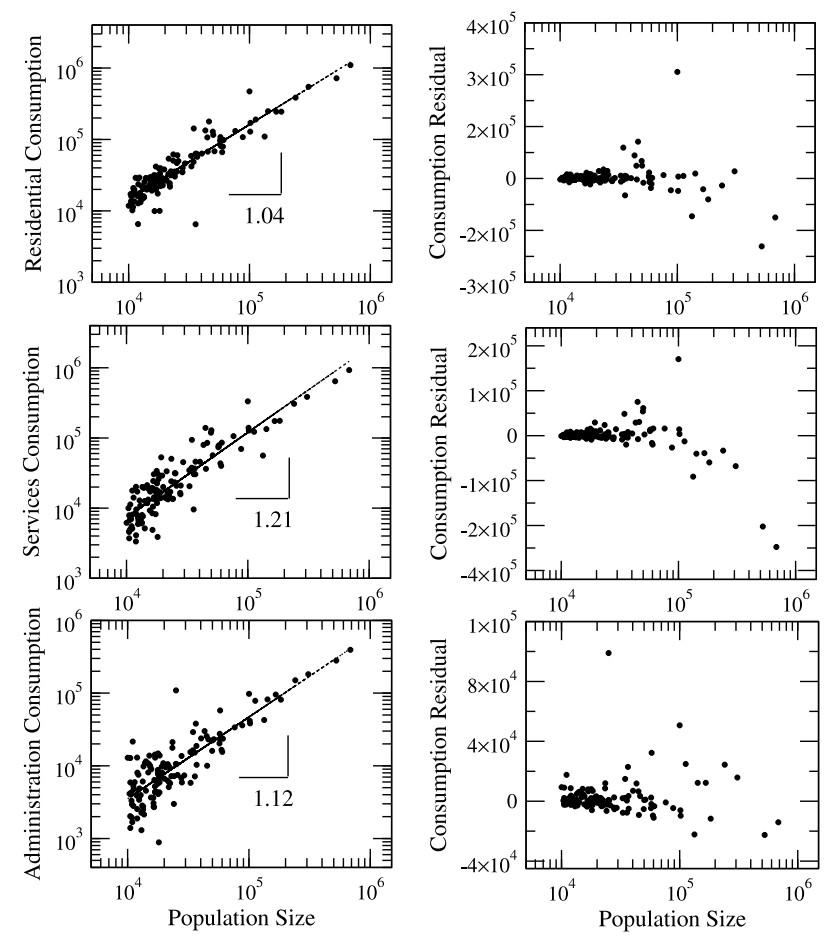

Fig. 2. Empirical allometric scaling relationships between electricity consumption and population size for residential, services and administration sectors (from top to bottom, plots on the left) and deviations from the predicted scaling behavior (plots on the right).

TABLE I

SCALING EXPONENTS WITH ADJUSTED R ${ }^{2}$ FOR CONSUMPTION SECTORS IN 2005 IN ANDALUCÍA

\begin{tabular}{|c|c|c|}
\hline Sector & $\beta$ & Adj. $\mathrm{R}^{2}$ \\
\hline \hline Primary & 0.42 & 0.04 \\
\hline Secondary & 1.07 & 0.28 \\
\hline Tertiary & 1.21 & 0.81 \\
\hline Residential & 1.04 & 0.85 \\
\hline Administration & 1.12 & 0.71 \\
\hline All & 1.06 & 0.75 \\
\hline
\end{tabular}

scaling behaviors for three different consumption sectors: administration (which includes educational, social and cultural facilities, public health, transport and institutions), household or residential, and finally tertiary sector or services. Numerical results are shown in Table I. As before, the figure also shows deviations from predicted scaling, measured as $d_{i}$. As we can see, $d_{i}$ grows with increasing population size. But a closer inspection of the deviation plots indicates a correlation pattern between household and tertiary consumption somehow different than that of administration consumption. That is, a city with large (small) household consumption is also energetically expensive (cheap) in the tertiary. But this trend is diluted in the administrative sector. Implications of this differences will be suggested in the conclusions section and investigated elsewhere. 

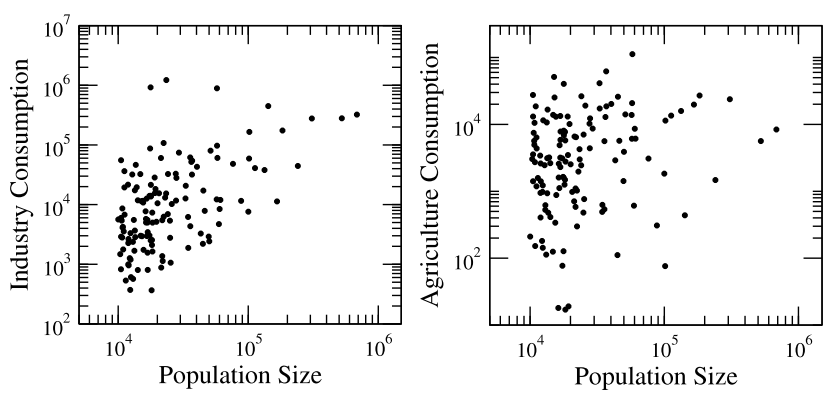

Fig. 3. Relationship between the electricity consumption in the industry (left) and agriculture (right) sectors show no clear scaling behavior.

\section{DISCUSSION}

Results presented so far show slightly superlinear scaling exponent for the aggregated electricity consumption in cities of southern Spain. This scaling behavior agrees with other similar results found in the literature for total and household electricity consumption [3], [5]. But variability arises when a non aggregated approach is considered. Residential consumption, for example, scales linearly with $\beta \approx 1$, as it would be expected from a variable directly related with population. Though on the other hand, administrative and tertiary sectors present clear superlinear scaling behaviors, both with $\beta>1$. This variability leads to the question of the magnitude of their values and the implications in the definition of a general picture of the urban scaling.

The fact that this path of inquiry and research is still in its infancy becomes still more obvious in Figure 3, where no scaling behavior whatsoever can be adjusted (i.e., low $\mathrm{R}^{2}$ ) for two particularly important consumption sectors. These are primary (agricultural) and secondary (industrial) sectors, where population must not be necessarily correlated with electricity consumption. Indeed, huge industrial facilities and agricultural infrastructures can be located near small villages or much more populated areas, depending on administrative, economic or financial advantages. The way these sectors could add up to the development of a detailed, if any, predictive theory for the scaling exponents $\beta$ is still unclear.

\section{CONCLUSION}

In this paper we have presented a brief analysis of electricity consumption patterns by means of their scaling behavior. Five different consuming sectors have been independently considered and analyzed, in contrast with only household [5] or household and total electricity consumption scalings analysis [3] found so far in the literature. This fact has given us the possibility to detect contrasting values in the scaling exponents, clearly attenuated in cumulative form. In doing so, while residential consumption scale almost linearly, superlinear patterns arise in tertiary and administrative consumptions. On the other hand, highly consuming sectors such as agriculture and industry, can not be scaled properly.

The way in which these results could be useful in order to develop a predictive theory for urban scaling in general, and electricity generation, distribution and consumption scaling in particular, is still vague and unclear. We believe that variability in $d_{i}$ and in the scaling exponent values suggest different underlying (a) social dynamics, (b) infrastructure designs or (c) an interactive and cumulative result of both influences. But different factors, such as finite size correction, statistical confidence interval considered or sample size, strongly affect the assumptions for modeling of scaling behavior and greatly complicate the finding of a suitable broad analytical framework [8]. We hope this research will ultimately lead to a better understanding of these inner dynamics and their relation with the evolution and structure of cities, as well as it has been achieved in the biology field.

\section{ACKNOWLEDGMENT}

The authors would like to thank Luís M. A. Bettencourt for useful conversations.

\section{REFERENCES}

[1] G. West and J. Brown, "The origin of allometric scaling laws in biology from genomes to ecosystems: towards a quantitative unifying theory of biological structure and organization," J. Exp. Biol., vol. 208, pp. 15751592, 2005.

[2] G. West, J. Brown, and B. Enquist, "A general model for the origin of allometric scaling laws in biology," Science, vol. 276, pp. 122-126, Apr. 1997.

[3] L. Bettencourt, J. Lobo, D. Helbing, C. Kuhnert, and G. West, "Growth, innovation, scaling, and the pace of life in cities," PNAS, vol. 104, no. 17, pp. 7301-7306, 2007.

[4] (2009) World Urbanization Prospects: The 2007 Revision Population Database. [Online]. Available: http://esa.un.org/unup/

[5] X. Zhu, A. Xiong, L. Li, M. Liu, and X. S. Chen, "Scaling law between urban electrical consumption and population in China," in Complex Sciences, ser. Lecture Notes of the Institute for Computer Sciences, Social Informatics and Telecommunications Engineering. Berlin: Springer Berlin Heidelberg, 2009, vol. 4.

[6] (2009) Instituto de Estadística de Andalucía. [Online]. Available: http://www.juntadeandalucia.es:9002/index.html

[7] L. M. A. Bettencourt, private communication, 2008.

[8] V. M. Savage, E. Deeds, and W. Fontana, "Sizing up allometric scaling theory," PLoS Comput. Biol., vol. 4, no. 9, 2008. 